\title{
The Structure of Membrane Preparations of the Fragmented Sarcoplasmic Reticulum after Freeze-Drying
}

\author{
Anneliese M. Lengsfeld and Wilhelm Hasselbach \\ Max-Planck-Institut für Medizinische Forschung, Abteilung Physiologie, \\ Heidelberg, Federal Republic of Germany
}

Received March 9, 1974

Summary. Different membrane preparations of the fragmented sarcoplasmic reticulum (FSR) were examined by means of the electron microscope 1. after freeze-drying, 2. after partial dehydration and 3. after standard dehydration and embedding. Prior to these comparative preparational procedures the isolated membranes were either left in their native state or incubated with $\mathrm{Hg}$-phenyl azoferritin or delipidated by digestion with phospholipase A and washing with albumin.

The freeze-drying technique yielded satisfactory results only when fixation was carried out with $\mathrm{OsO}_{4}$ vapour after accomplishing the drying. Even then, good preservation of the vesicular and membrane structure could -if at all - only be obtained in the very surface area of the pellet droplets, maximally up to a depth of $2-3 \mu \mathrm{m}$.

The average vesicle diameter of freeze-dried sarcoplasmic membranes measures slightly less than that of chemically dehydrated membranes, particularly clearly demonstrated in delipidated preparations. The membrane structure itself, however, is thicker in freeze-dried than in chemically dried material. A peculiarity of freeze-dried untreated and ferritin incubated membranes is that the usually light inner layer of the unit membrane is comparatively darker than in the corresponding chemically dehydrated preparations and than in all delipidated preparations, thus giving rise to a more compact membrane appearance of these freezedried FSR vesicles.

While in sections of ferritin decorated, chemically dehydrated preparations the ferritin particles are arranged like a chain of pearls outside around the membrane, the ferritin particles are located in freeze-dried material immediately on the outer dense line of the vesicular membrane.

\section{Introduction}

Numerous substances are partially or completely extracted from biological material during routine fixation, dehydration and embedding procedure for electron microscopy. This holds particularly true for water soluble components such as sugars as well as for substances soluble in alcohol or acetone such as lipids (Stein and Stein, 1971). According to Stein and Stein (1971) phospholipids however can be nearly completely retained when the tissue is partially dehydrated (Stein, Scow and Stein, 1970). Another method for preserving water, acetone or alcohol soluble components for electron microscopic examination would be the freeze-drying technique used by Stirling and Kinter (1967) and improved by Eckert (1969).

The intention of this investigation was to find out, whether (by using the techniques of freeze-drying and subsequent embedding) the extraction of lipids from biological membranes can be avoided or reduced when the chemical dehydration through a graded series of acetones or alcohols is replaced by drying in high 
vacuum. We were interested to see, whether and what kind of morphological differences in the membrane structure might be found after the preparations have been freeze-dried, partially dehydrated and routinely embedded. These madifications should give rise to different degrees of lipid extraction.

We studied isolated sarcoplasmic membranes (FSR) of rabbit skeletal muscle, which have been widely examined by means of the electron microscope in a number of investigations, using standard embedding as well as negative staining and freeze etching techniques (Agostini and Drabikowski, 1969; Agostini and Hasselbach, 1971a, 1971 b, 1973; Deamer and Baskin, 1969; Hasselbach and Elfvin, 1967 ; Ikemoto et al., 1968). After treating the isolated membranes differently before either freeze-drying or partial dehydration or routine embedding the resulting structures were compared. It was not possible, however, to examine the lipid extraction from the membrane material during fixation, dehydration and embedding by radioactivity measurements, because small amounts of the membrane material are continously lost during each step of the preparation procedure.

\section{Materials and Methods \\ Fragmented Sarcoplasmic Reticulum}

FSR vesicles were obtained from skeletal muscles of adult rabbits essentially according to Hasselbach and Makinose (1963).

\section{Treatment of FSR Vesicles prior to Electron Microscopic Preparation}

The following membrane preparations were used for comparative freeze-drying, partial dehydration and standard embedding procedure:

1. Untreated FSR vesicles.

2. Hg-phenylazoferritin Decorated Sarcoplasmic Membranes. The Hg-phenyl azoferritin was obtained according to Hasselbach and Elfvin (1967) with small modification as described by Lengsfeld and Hasselbach (1971).

The incubation of the membranes with $\mathrm{Hg}$-phenylazoferritin was carried out according to Hasselbach and Elfvin (1967).

3. Delipidated FSR Vesicles. Sarcoplasmic membranes were delipidated essentially according to Fiehn and Hasselbach (1970) by digestion with phospholipase A and subsequent washing with albumin. $1 \mathrm{mg}$ phospholipase $\mathrm{A} / 100 \mathrm{mg}$ membrane protein was added to a solution of $0.1 \mathrm{M} \mathrm{KCl}, 0.02 \mathrm{M}$ Tris, $\mathrm{pH} 8.5,5 \mathrm{mM} \mathrm{CaCl} 2$. The membrane-phospholipase mixture was stirred for 2 hours at room temperature. Then $4 \%$ albumin was added and after stirring for another 10-15 min the membranes were centrifuged for $40 \mathrm{~min}$ at $30000 \times \mathrm{g}$ (Sorvall Type SS-1, highest speed). Subsequently the membranes were washed 1) either a second time with $1-2 \%$ albumin in $0.1 \mathrm{M} \mathrm{KCl}$, centrifugation $30 \mathrm{~min}$ at $30000 \times \mathrm{g}$, or 2) twice with $0.1 \mathrm{M}$ ammonium acetate, $\mathrm{pH} 7.2$, centrifugations 1 hour resp. $30 \mathrm{~min}$ at $30000 \times \mathrm{g}$, or 3) twice with $0.1 \mathrm{M} \mathrm{KCl} / 0.02 \mathrm{M}$ Tris, centrifugations 1 hour resp. $30 \mathrm{~min}$ at $30000 \times \mathrm{g}$.

\section{Electron Microscopy}

Freeze-Drying. For freeze-drying the membrane vesicles, originally suspended in $0.1 \mathrm{M}$ $\mathrm{KCl}$ were washed either once or twice with distilled water or twice with $0.1 \mathrm{M}$ ammonium acetate, pH 7.2 (centrif ugations $30-45 \mathrm{~min}$ at $30000 \times \mathrm{g}$, Sorvall, Type SS-1, highest speed) in order to avoid $\mathrm{KCl}$ crystals in the freeze-dried embedded membrane material. Similarly, the $\mathrm{Hg}$-phenylazoferritin incubated membranes after the third washing with $0.1 \mathrm{M} \mathrm{KCl} / 0.05 \mathrm{M}$ $\mathrm{K}_{2} \mathrm{HPO}_{4}$, were washed either twice with distilled water or twice with $0.1 \mathrm{M}$ ammonium acetate, centrifugations once $15 \mathrm{~min}$ at $4600 \times \mathrm{g}$, once $30 \mathrm{~min}$ at $30000 \times \mathrm{g}$ (Sorvall). All procedures were performed at $2-4^{\circ} \mathrm{C}$. 
Freeze-drying and subsequent embedding of the FSR vesicles were carried out according to Eckert (1969), some modifications were introduced. Prior to deep-freezing the sarcoplasmic membranes were spun down to obtain loose pellets. By dipping the tips of thin glass needles or capillaries into the pellet material, small droplet-shaped samples of a diameter of $1 / 2-1 \mathrm{~mm}$ were prepared, which, together with the glass needles were carried over into tubes with liquid propane of $-180^{\circ} \mathrm{C}$ for rapid deep-freezing. Copper wire as applied by Eckert for tissue blocks could not be used, since the pellet material did not stick to it. The test tubes were then attached to a freeze-drying apparatus constructed as described by Eckert. Two samples could be dried at once. The preparations were dried at $10^{-4}-10^{-5}$ Torr and at $-80^{\circ} \mathrm{C}$. It proved necessary to dry the material for 6-7 days at this low temperature. Then the temperature was slowly raised to $-40^{\circ} \mathrm{C}$, at which temperature the preparation was kept for 15-24 hours. Subsequently the temperature was raised to room temperature over a period of about 4 hours.

Fixation was carried out with osmium tetroxide vapour for $2-2^{1} / 2$ hours at room temperature. In modification of Eckert's description, the ampoules containing the $\mathrm{Os}_{4} \mathrm{O}_{4}$ crystals were put directly into the test tubes containing the dried FSR vesicles, to shorten the diffusion distance of $\mathrm{OsO}_{4}$. For this procedure the tube with the dried membranes had to be removed from the vacuum apparatus for a short moment, but immediately after fixing the $\mathrm{OsO}_{4}$ containing ampoule it was again evacuated to $10^{-2}-10^{-3}$ Torr and left under vacuum for the fixation period. This short interruption of vacuum apparently did not effect the dried membrane material. After fixation the $\mathrm{Os}_{4}$ containing ampoule was removed during another quick interruption of vacuum, and subsequently the excess $\mathrm{OsO}_{4}$ was sublimed into the cold trap at $10^{-4}-10^{-5}$ Torr for $30-60 \mathrm{~min}$.

For embedding the dried and fixed membrane material, partly still adhering to the glass needles as droplets, partly broken apart into smaller pieces, was quickly carried over into flat dishes containing Epon 812. After allowing the Epon to suck the dried material for $2-3$ days, polymerization took place at $60^{\circ} \mathrm{C}$ for 48 hours.

In these experiments we did not only use sarcoplasmic membrane material unfixed before freezing and drying. For comparison we also used FSR vesicles fixed prior to freeze-drying, either with an $\mathrm{Os} / \mathrm{Cr}$ mixture, containing $2 \% \mathrm{OsO}_{4}$ and $2 \% \mathrm{~K}_{2} \mathrm{Cr}_{2} \mathrm{O}_{7}, \mathrm{pH} 7.2$ (untreated sarcoplasmic membranes), or with $3 \%$ glutaraldehyde $(G A)$ in Sörensen phos. phate buffer, $\mathrm{pH}$ 7.2, and postfixed with the above Os/Cr mixture (Hg-phenyl azoferritin incubated membranes). Before deep-freezing the fixed membranes were washed several times with distilled water. The freeze-drying and embedding procedure was accomplished as described above.

Partial Dehydration. Partial dehydration and embedding in Epon were carried out sesentially according to Stein, Scow and Stein (1970). The untreated FSR vesicles suspended in $0.02 \mathrm{M}$ Tris/0.1 M KCl were fixed for $45 \mathrm{~min}$ in $5 \% \mathrm{GA}$ in Sörensen phosphate buffer, $\mathrm{pH}$ 7.2, washed overnight with buffer and postfixed in two ehanges of the above described Os/Cr mixture (Wohlfahrt-Bottermann, 1957) for 15 min each. After washing with tyrode for 1 hour the membrane material was stained in block for 45 min with $1 \%$ phosphotungtic acid (PTA) and $0.5 \%$ uranyl acetate in $70 \%$ ethanol (Wohlfahrt-Bottermann 1957). The subsequent procedure followed Stein et al. (1970).

In the same way fixation and embedding were carried out with the Hg-phenyl azoferritin incubated sarcoplasmic membranes after they had been washed three times with $0.1 \mathrm{M} \mathrm{KCl} /$ $0.05 \mathrm{M} \mathrm{Na}{ }_{2} \mathrm{HPO}_{4}$, and with delipidated vesicles after they had been washed twice with $0.1 \mathrm{M} \mathrm{KCl} / 0.02 \mathrm{M}$ Tris.

Routine Fixation and Embedding. The untreated sarcoplasmic membranes suspended either in $0.1 \mathrm{M} \mathrm{KCl}$ or in $0.1 \mathrm{M}$ ammonium acetate, and the delipidated vesicles suspended either in $1 \%$ albumin containing $0.1 \mathrm{M} \mathrm{KCl}$ or in $0.1 \mathrm{M}$ ammonium acetate, $\mathrm{pH} 7.2$, or in $0.1 \mathrm{M} \mathrm{KCl} / 0.02 \mathrm{M}$ Tris, were fixed with the Os/Cr mixture for $30-45 \mathrm{~min}$, while the $\mathrm{Hg}$-phenyl azoferritin incubated membranes were fixed with $3 \%$ GA in Sörensen phosphate buffer, $\mathrm{pH} 7.2$, and postfixed with the Os/Cr solution. Dehydration was carried out in a graded series of acetones, with $1 \% \mathrm{PTA}$ and $0.5 \%$ uranyl acetate in the $70 \%$ acetone for block staining. Epon was used as embedding medium.

Sections were cut with glass knives on an LKB Ultrotome III, LKB Productors Stockholm, and poststained with lead citrate (Venable and Coggeshall 1965) or double-stained with 
uranyl acetate and lead citrate. Electron micrographs were taken with a Siemens EM 101, equipped with an anticontamination device, using a double condensor illumination and operating at $80 \mathrm{kV}$.

\section{Results}

\section{Freeze-dried Membrane Material}

The structure of the sarcoplasmic vesicles was well preserved only in areas, which were located in the very narrow surface zone of the freeze-dried dropletshaped pellet material. The thickness of this well preserved layer did not exceed a few $\mu \mathrm{m}$. It was therefore important to choose for sectioning only the surface zone of the dried membrane droplets. Luckily, even when these were broken apart prior to or during embedding, the original surface site could be distinguished in the polymerized flat Epon blocks by its rounded smooth appearance, when examined under a stereo microscope.

Satisfactory results were obtained only when the membrane material was first freeze-dried and subsequently fixed with $\mathrm{OsO}_{4}$ vapour, but not when fixation took place prior to deep-freezing and drying. In the latter case-no micrographs are included-the sarcoplasmic membrane vesicles, largely sticking together, have been nearly completely disrupted during the freeze-drying procedure. Instead of closed vesicles nearly only open membrane fragments are discernable. However, some of these show a distinct trilamellar appearance with a width of $90-110 \AA$, the inner light layer being remarkably lighter then the electron dense outer lines. In addition, five-lamellar membrane structures with a thickness of 150-180 $\AA$ can be observed, probably originating from adjacent membrane vesicles, whose membranes have intimately stuck together.

Plate I shows thin sections of sarcoplasmic membrane material after freezedrying and subsequent fixation with $\mathrm{OsO}_{4}$ vapour. Membrane preparations pretreated in different ways did not give equally good results by the freezedrying technique. Cross sections of the surface layer of the pellet droplets of untreated (Fig. 1), Hg-phenylazoferritin incubated (Fig. 2), and delipidated sarcoplasmic membranes (Fig. 3) are presented for comparison in the same scale, at low (a) and higher (b) magnification.

The best results with untreated FSR membranes were obtained, when the vesicle preparation was washed with distilled water before deep-freezing (Fig. 1). Washing with ammonium acetate did not improve structural preservation, though the results were quite satisfactory, too. The vesicular and membrane structure is well preserved up to a distance of $2-3 \mu \mathrm{m}$ from the surface of the pellet droplets (Fig. 1). Open vesicles are comparatively seldom in the outer layer of $1-1^{1 / 2} \mu \mathrm{m}$, but their frequency increases with the distance from the surface. Corresponding to the higher frequency of disruptered vesicles towards the inside of the pellet droplets, the vesicles show a progressing tendency to clot together. Sometimes they are forming strands of membrane material separated by areas of membrane free embedding medium, the individual vesicles then not being ruptured to the same extent, as seen in Fig. 1a, upper part.

When the untreated membrane preparation was washed with ammonium acetate before freeze-drying, more open vesicles were observed in the very outer 

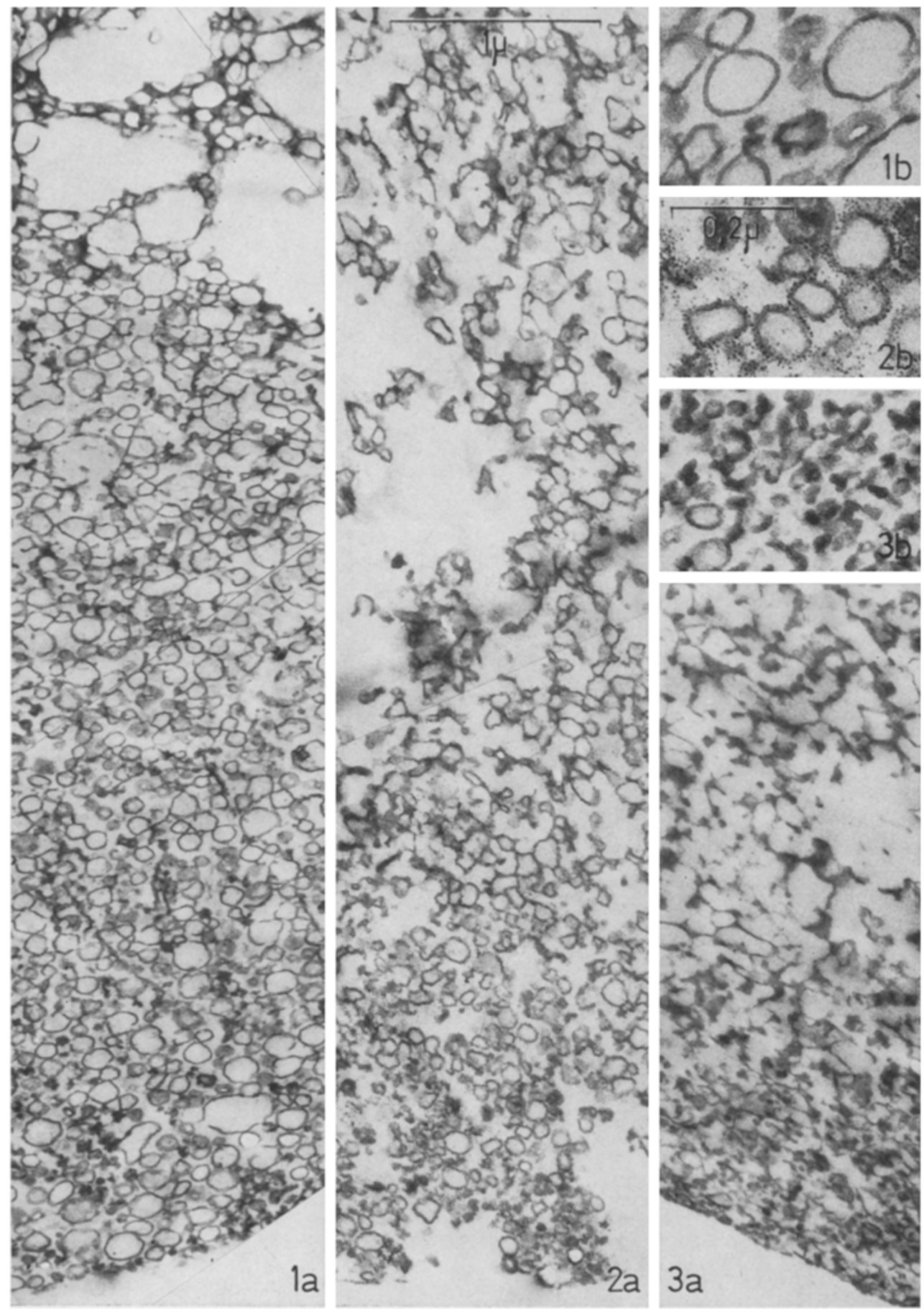

Figs. 1-3. Cross sections through the surface area of freeze-dried pellet droplets of isolated sarcoplasmic membranes pretreated in different ways: Fig. 1 native FSR vesicles, Fig. 2 Hgphenyl azoferritin incubated preparation, Fig. 3 delipidated membranes. Good preservation of the vesicular and membrane structure took place only in the very surface zone of the pellet droplets, at the highest to a depth of $2-3 \mu$ (Fig. 1a). Fixation with $\mathrm{O}_{\mathrm{s}} \mathrm{O}_{4}$ vapour; a $29760: 1$, b $83700: 1$ 

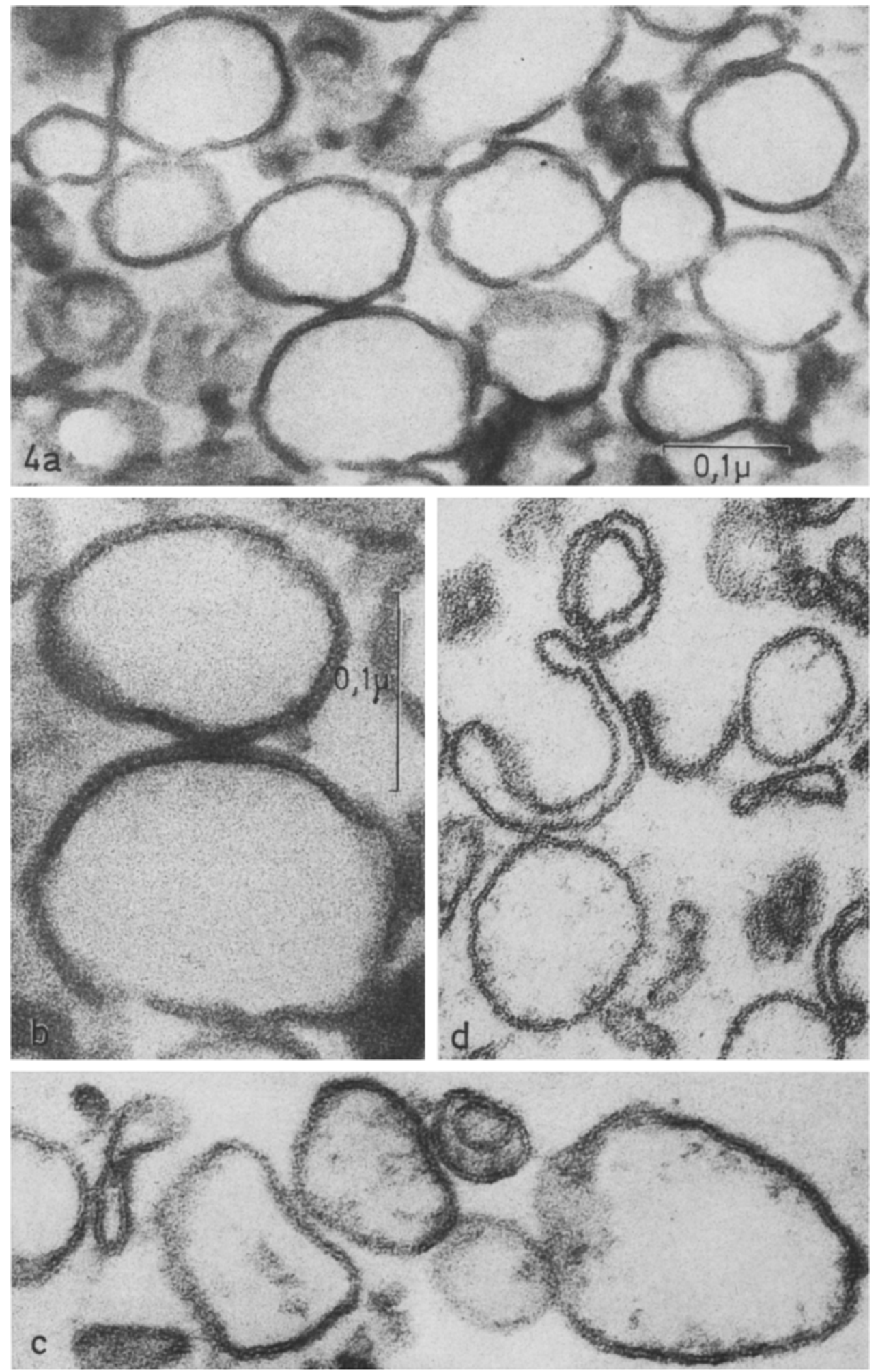

Fig. $4 a-d$ 
layer of the droplets, and sticking together and disrupturing of the vesicles occurred more often, while the membrane structure itself was well preserved.

Ferritin labelled membrane preparations displayed good structural preservation after freeze-drying only, when washed with ammonium acetate before deepfreezing (Fig. 2a). The well preserved surface layer of the pellet droplets was normally only about $11 / 2-2 \mu \mathrm{m}$ thick. In deeper layers of the pellet, agglutination and disrupturing occurred more often than in untreated membrane vesicles.

Acceptable results of delipidated sarcoplasmic membranes were obtained, when the preparations were washed either with ammonium acetate or with $0.1 \mathrm{M}$ $\mathrm{KCl} / 0.02 \mathrm{M}$ Tris; the latter proved more satisfactory. However, in comparison with freeze-dried untreated and $\mathrm{Hg}$-phenylazoferritin labelled membrane preparations, the yield of well preserved structures of freeze-dried delipidated sarcoplasmic membrane preparations was quite low. As shown in Fig. 3 a the vesicular and membrane structure of the delipidated FSR material is fairly well preserved only till a depth of about $1 / 2 \mu \mathrm{m}$. Further inside the droplet, even at a distance of only $1-2 \mu \mathrm{m}$, the pellet material is disruptured extensively. Good structural preservation of the delipidated membranes until a depth of $1-1^{1 / 2} \mu \mathrm{m}$ from the droplet surface could be found only occasionally.

As to the size of the vesicles of the different preparations, the diameter of untreated and ferritin incubated FSR vesicles usually ranges from $0.06-0.2 \mu \mathrm{m}$, the ferritin vesicles being slightly smaller than the untreated ones. The delipidated membrane vesicles are extremely small, having an average diameter of only 0.02-0.04 $\mu \mathrm{m}$ (compare (Figs. $1 \mathrm{~b}-3 \mathrm{~b}$ ).

\section{Untreated Sarcoplasmic Membrane Preparations}

Sections comparing native sarcoplasmic membrane preparations after freezedrying (a and b), partial dehydration (c) and standard embedding procedure (d) are presented in figure 4 ( $a, c$ and $d$ same magnification). The vesicle diameter of native FSR membranes ranges normally from $0.06-0.4 \mu \mathrm{m}$; the average vesicle diameter, however, is slightly smaller in freeze-dried than in partially or standardly dehydrated material (see Table 1). The membrane thickness, on the contrary, seems to be enlarged when chemical dehydration is abbreviated (partial dehydration) or avoided (freeze-drying), as seen in Table 1.

A remarkable peculiarity of the freeze-dried membranes is the relatively electron dense inner layer of the trilamellar unit membrane (in comparison with partially dehydrated and routinely embedded material). Though the outer dense lines are elearly more electron opaque than the grayish middle layer, the freezedried membranes thus display a more compact appearance (Fig. $4 \mathrm{a}$ and b), which

Fig. 4a- d. Sectioned pellet material of untreated FSR membranes after freeze-drying and subsequent fixation with $\mathrm{OSO}_{4}$ vapour (a and b), after partial dehydration (c) and after standard dehydration (d). The vesicular diameter of the freeze-dried membranes is slightly smaller than in the chemically dehydrated preparations, while the membrane structure itself is thicker after freeze-drying. Note the grayish middle layer of freeze-dried membranes (a and b), which is comparatively darker than in partially (c) and routinely (d) dehydrated membranes. a, c, d 180000:1, b 300000:1 

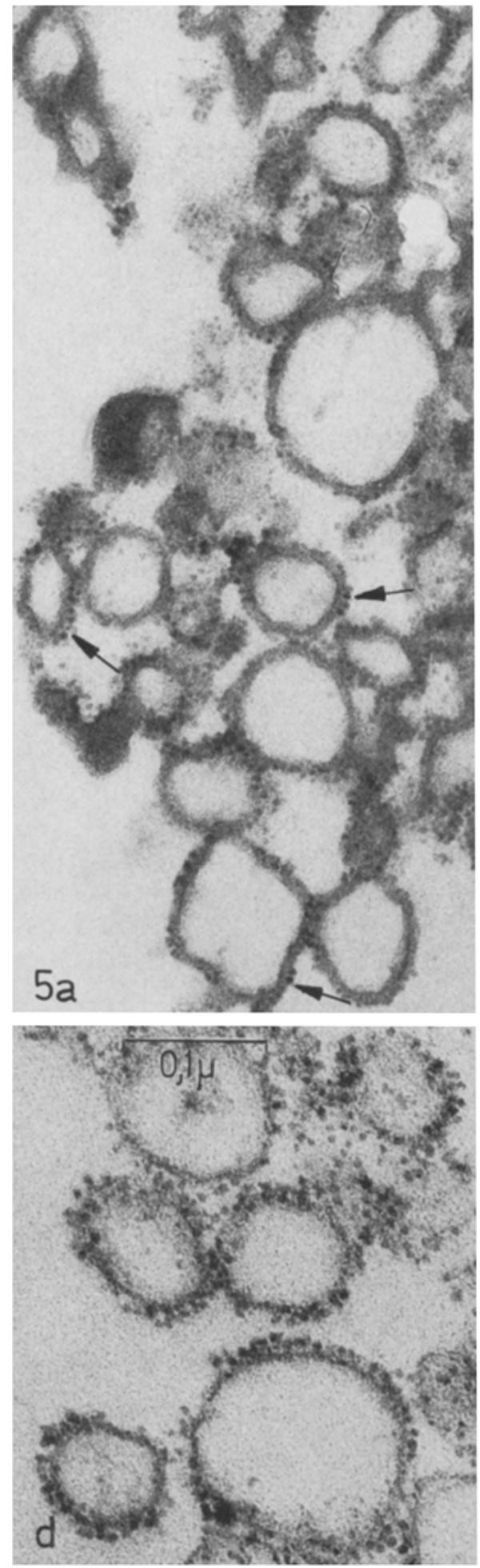
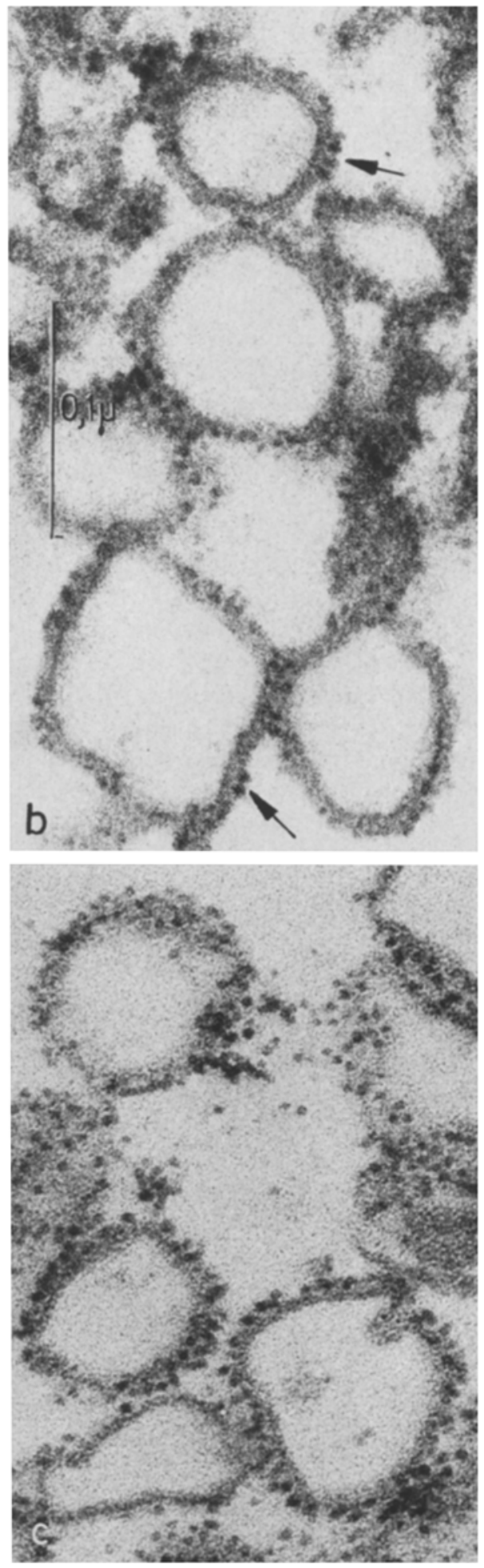
Table 1. Average vesicle diameter and membrane thickness of the different membrane preparations after freeze-drying (FD), partial dehydration (PD) and standard embedding (SE)

\begin{tabular}{lll}
\hline & $\begin{array}{l}\text { Average vesicle } \\
\text { diameter } \\
(\mu)\end{array}$ & $\begin{array}{l}\text { Membrane } \\
\text { thickness } \\
(\AA)\end{array}$ \\
\hline I. Native FSR preparations & & \\
1. after freeze-drying & $0.07-0.2$ & $75-90$ \\
2. after partial dehydration & $0.1-0.25$ & $65-75$ \\
3. after standard embedding & $0.1-0.25$ & $57-65$ \\
II. Ferritin labelled sarcoplasmic membranes & \\
1. after freeze-drying & $0.06-0.11$ & $65-80$ \\
2. after partial dehydration & $0.1-0.25$ & $55-65$ \\
3. after standard embedding & $0.08-0.2$ & $50-55$ \\
III. Delipidated sarcoplasmic membranes & & \\
1. after freeze-drying & $0.02-0.035$ & $65-80$ \\
2. after partial dehydration & $0.04-0.06$ & $60-70$ \\
3. after routine embedding & $0.04-0.06$ & $55-65$ \\
\hline
\end{tabular}

definitely differs from the distinct and very contrastful dark-light-dark triplelayer aspect of the partially dehydrated and routinely embedded FSR membranes (Fig. $4 \mathrm{c}$ and $4 \mathrm{~d}$ ).

\section{Hg-phenylazoferritin Incubated Membrane Preparations}

Fig. 5 shows $\mathrm{Hg}$-phenylazoferritin incubated FSR vesicle preparations after freeze-drying and subsequent fixation with $\mathrm{OsO}_{4}$ vapour (a and b), after partial dehydration (c) and after routine embedding procedure (d) (a, c, d same magnification).

As observed in native membrane preparations freeze-dried ferritin decorated FSR preparations are characterized by a slightly smaller vesicular diameter and a larger membrane width than the corresponding chemically dehydrated ones (compare Table 1).

Similar as in the native FSR preparations the middle layer of freeze-dried ferritin decorated membranes (Fig. 5a, b) is comparatively darker than in the chemically dehydrated ones (Fig. $5 \mathrm{c}, \mathrm{d}$ ).

Concerning the ferritin binding to the membranes, a difference can be observed. between the freeze-dried and chemically dried material. While in the sectioned partially dehydrated (Fig.5c) and standardly embedded FSR preparations (Fig. 5d)

Fig. 5a-d. Hg-phenylazoferritin incubated membrane preparations, after freeze-drying and subsequent fixation with $\mathrm{OsO}_{4}$ vapour (a and b), after partial dehydration (c) and after rontine dehydration and embedding (d). Note the difference in ferritin binding: While in sections of chemically dehydrated FSR preparations the ferritin particles are arranged like a chain of pearls outside around the membranes ( $c$ and d), they are located in freeze-dried material immediately on the outer dense line of the membrane, thus partly overlapping the membrane itself ( $a$ and $b$, arrows). a, c, d 180000:1, b 300000:1 

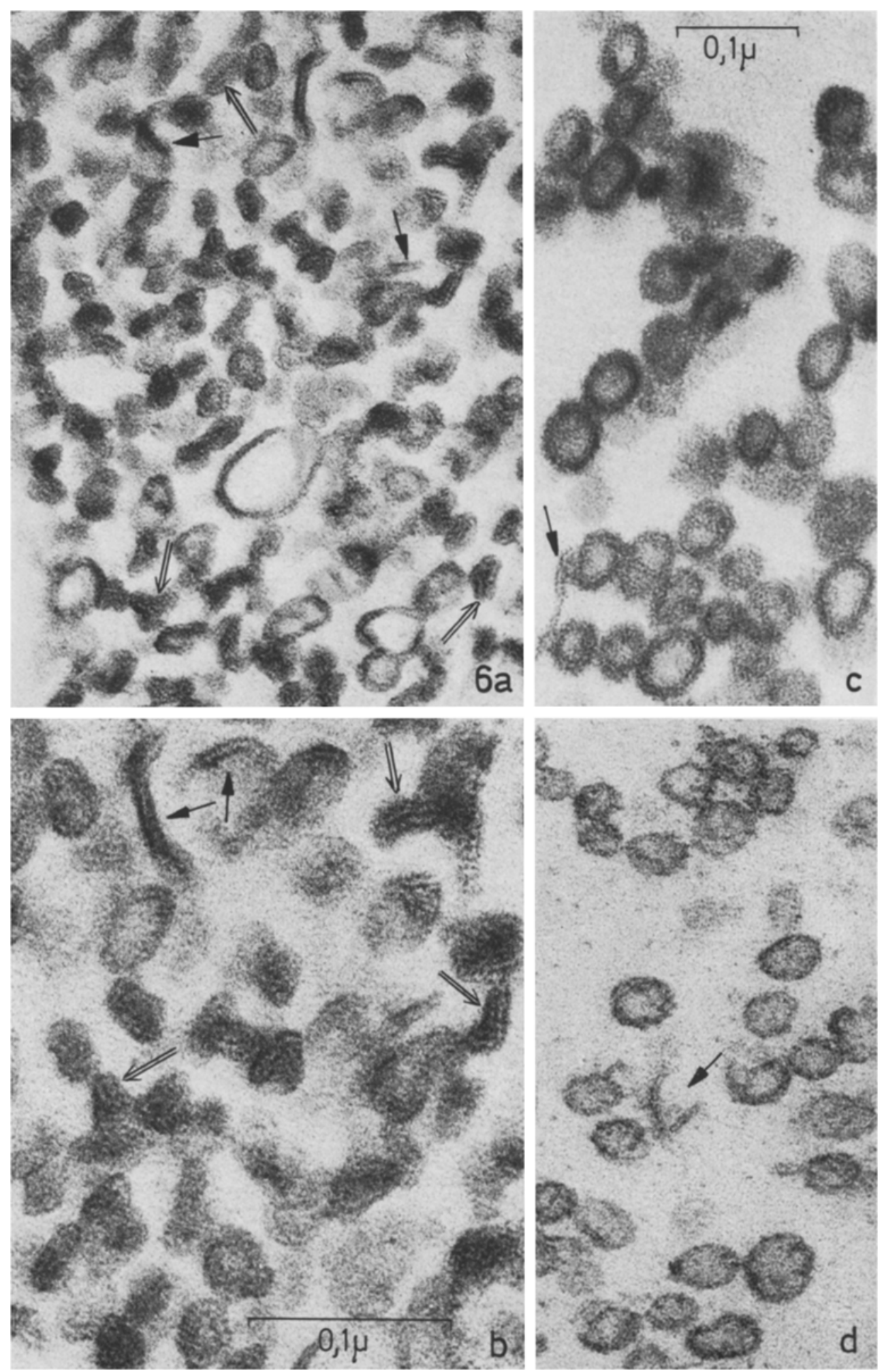
the ferritin particles are arranged like a chain of pearls outside around the membranes, the ferritin particles are located in the sectioned freeze-dried material directly on the outer dense line of the membrane structure itself, thus partly overlapping the membrane itself (Fig. 5 a and b, arrows).

\section{Delipidated Sarcoplasmic Membranes}

Comparative sections of phospholipase A digested sarcoplasmic membrane preparations are shown in Fig. 6 after freeze-drying (a and b), after partial dehydration (c) and after standard embedding (d) (a, c, d same magnification).

Delipidated FSR preparations consist mostly of closed vesicles, but open membrane fragments (Fig. 6, arrows) are more frequently found than in native isolated sarcoplasmic membranes. As already reported by Agostini and Hasselbach $(1971 \mathrm{a}, 1973)$, the vesicle size of delipidated membranes is considerably smaller than in native preparations (compare Fig. $6 \mathrm{c}$ and $\mathrm{d}$ with Figs. 4 and 5). However, compared to these, the vesicular diameter of freeze-dried phospholipase A digested membranes is further diminished measuring mostly only $0.02-0.035 \mu \mathrm{m}$ (compare Fig. 6a, c, d and Table 1). The freeze-dried vesicles have often more or less completely collapsed, thus giving rise to five-lamellar membrane structures (Fig. 6a and b, double arrows).

As in native and in ferritin labelled FSR preparations the membrane width of freeze-dried delipidated membranes is larger than in chemically dehydrated delipidated preparations (see Table 1).

When compared to freeze-dried untreated and ferritin decorated membranes, the middle layer of the freeze-dried delipidated membranes is markedly lighter than the outer electron opaque layers, thus giving rise to the contrastful darklight-dark three layered appearance usually observed in chemically dehydrated membranes.

\section{Discussion}

The freeze-drying of isolated sarcoplasmic membranes yields satisfactory results only when fixation was carried out with $\mathrm{OsO}_{4}$ vapour after the drying procedure, but not when fixation took place before deep-freezing. Furthermore, the vesicular and membrane structure of the FSR preparations was well preserved only in the very surface layer of the pellet droplets, maximally up to a depth of $2-3 \mu \mathrm{m}$. Further inside the droplets the vesicles were highly disruptured; the degree of disrupture increased with the distance from the surface.

When compared to Eckert's (1969) good structural preservation of freezedried tissues, our experiments with pellet material thus proved to be less successful.

Fig. 6a-d. Sectioned pellet material of phospholipase A digested FSR, after freeze-drying and subsequent fixation with $\mathrm{OsO}_{4}$ vapour ( $a$ and $b$ ), after partial dehydration (c) and after standard dehydration (d). The vesicle diameter of delipidated membranes being considerably smaller than in native FSR preparations (comp. $\mathrm{c}$ and $\mathrm{d}$ to $4 \mathrm{c}$ and $\mathrm{d}$ ), is especially tiny after freeze-drying ( $a$ and b). Arrows: open membrane fragments; double arrows in $a$ and $b$ : collapsed vesicles giving rise to five-lamellar membrane structures, a, c, d 180000:1, b 300000:1 
Apparently the application of the freeze-drying technique to pellet material is more difficult than for tissues.

However, it is questionable, whether the high degree of disrupture in deeper layers of the freeze-dried pellet droplets is caused by the freeze-drying procedure itself or whether these damages are not more likely introduced in a later stage of the fixation and/or embedding procedure. The following observations seem to indicate, that the disruptures are probably not primarily due to the freezedrying procedure itself. When lyophilized FSR vesicles, which show a collapsed aspect after fixation with $\mathrm{OsO}_{4}$ vapour and subsequent embedding, are resuspended in $0.1 \mathrm{M} \mathrm{KCl}$, they regain their usual vesicular appearance, so that sectioned pellets of fresh and resuspended lyophilized preparations hardly show any differences (unpublished data). Similar results were reported by Sreter, Ikemoto and Gergely (1970) with negatively stained fresh and reconstituted lyophilized FSR preparations.

The average diameter of freeze-dried membrane vesicles measures altogether slightly less than the average vesicle diameter of the corresponding FSR preparations after partial dehydration and routine embedding. This is most clearly demonstrated by the freeze-dried delipidated membrane preparations, whose vesicles are extremely tiny. Apparently the drying in high vacuum has caused shrinkage of the vesicle diameter by the removal of water out of the vesicular membranes, while during chemical dehydration, on the contrary, the exchange of water against acetone or ethanol largely preserves the original vesicle size.

Four facts indicate that less lipids are removed from the sarcoplasmic membranes when the preparations are freeze-dried: 1 . The membrane structure of freeze-dried untreated and ferritin decorated FSR preparations is markedly thicker than in routinely embedded material. 2. In freeze-dried native and ferritin decorated sarcoplasmic membranes the middle layer of the trilamellar unit membrane is comparatively darker than in the corresponding routinely embedded preparations. 3. These structural differences between freeze-dried and chemically dried preparations are much less pronounced, when the membranes have been enzymatically delipidated. 4 . The thickness of partially dehydrated membranes is in between that of freeze-dried and routinely embedded material.

The latter finding implies that partial dehydration does not only considerably reduce the extraction of neutral lipids from the tissues, as reported by Stein and Stein (1971) and Stein et al. (1970), but that it also improves the retention of phospholipids and neutral lipids in isolated sarcoplasmic membranes.

However, a direct proof of these conclusions would require tracing of lipid extraction with radioactive labelled lipids. But it is difficult to apply this method to pelleted membranes, since small amounts of the material are normally lost in each step of the fixation, washing, dehydration and embedding procedure, so that radioactivity measured in the discarded solutions would not only originate from extracted lipids, but from lost membrane material as well.

Our results seem to be at variance with those of Cope and Williams (1969a, $1969 \mathrm{~b}$ ), who found the lowest loss of two labelled glycerophosphatides from rat liver after fixation with $\mathrm{OsO}_{4}$ and subsequent dehydration in ethanols and embedding in Araldite, while all other procedure. including freeze-drying and freeze substitution, led to rather extensive loss of labelled phospholipid. However, 
these authors did not perform any fixation of the freeze-dried tissue and used only glutaraldehyde fixation for the freeze-substituted material before embedding; moreover, they used methacrylate for embedding, a medium which is known to dissolve lipids (Cope and Williams, 1968).

Concerning the chemical structure of the membrane itself, i.e. the distribution of lipids and proteins in the membrane, the following facts indicate a location of the lipids mainly in the middle layer of the membrane, while the proteins or at least those parts of the protein molecules which stain with $\mathrm{OsO}_{4}$, are arranged preferentially in the outside layers of the membrane structure: 1 . The-probably partially lipid extracted-chemically dehydrated native and ferritin labelled sarcoplasmic membranes as well as the enzymatically delipidated membranes after freeze-drying as well as after routine embedding show a distinct light inner layer of the unit membrane, thus displaying the usual dark-light-dark triple layered membrane aspect. 2 . In comparison to these the freeze-dried native and ferritin decorated membranes, which have retained more lipids have a relatively electron dense inner layer, thus giving rise to a more compact membrane appearance.

It would be difficult to explain our results in accordance to Mühlethaler's newly developed membrane model, which has been derived from freeze-etching experiments. This model shows the protein molecules located mainly inside the lipid bilayer, but partly extending through the lipid layer and thus reaching the membrane surface on one side or on both sides (Mühlethaler, 1972).

For the interpretation of our findings we need not necessarily assume a modified Danielli membrane model, but a somewhat modified fluid mosaic model of membrane structure as proposed by Singer and Nicolson (1972), could also be taken as a base, particularly as new biochemical data of Migala, Agostini and Hasselbach (1973) could be interpreted in terms of this model.

As far as the observed differences in the location of the bound ferritin particles at the freeze-dried membranes and the chemically dehydrated membranes are concerned, they seem to indicate that some kind of configurational change of the membrane structure has taken place either during freeze-drying and subsequent fixation with $\mathrm{OsO}_{4}$ vapour or during the standard fixation and chemical dehydration procedure. As a consequence the ferritin particles have either penetrated more deeply the membrane (freeze-dried preparations) or have been expelled from the membrane (chemically dehydrated material).

Yet it cannot be decided at present, which of the observed configurations is the original one and which the by fixation and dehydration altered one, or whether possibly both arrangements have to some extent arisen artificially by the fixation and drying procedure.

Alterations in the location of bound ferritin particles at sarcoplasmic membranes have also been reported by Agostini and Hasselbach (1973) after delipidation of the membranes with phospholipase A. These findings are interpreted as an indication "that some changes in the configuration of membrane protein" (p. 105) have possibly occurred when the lipid composition was altered.

We wish to thank Mrs. Elizabeth Alexander-Jelinek for her excellent and invaluable technical assistance. 


\section{References}

Agostini, B., Drabikowski, W.: Morphological changes in the fragmented sarcoplasmic reticulum after oleate treatment. J. submicr. Cytol. 1, 207-214 (1969)

Agostini, B., Hasselbach, W.: Correlazioni strutturali, eitochimiche ed enzimatiche nelle membrane del reticulo sarcoplasmatico. Atti II Simposio Nationale sui Metodi: Enzimatici nella Diagnostica Clinica. Conegliano Veneto 1970 In: Quad. Sclavo Diagn. 7, 406-428 (1971a)

Agostini, B., Hasselbach, W.: Electron cytochemistry of calcium uptake in the fragmented sarcoplasmic reticulum. Histochemie 28, 55-67 (1971b)

Agostini, B., Hasselbach, W.: Structural and cytochemical studies on fragmented sarcoplasmic reticulum following treatment with various lipolytic enzymes. In: Basic research in myology. Proceedings of the 2nd internat. Congress on musele diseases. Perth Nov. 71, ed. by B. A. Kakulas. Amsterdam; Excerpta Medica; New York: American Elsevier Comp., Inc. 1973

Cope, G. H., Williams, M. A.: Quantitative studies on neutral lipid preservation in electron microscopy. J. roy, micr. Soc. 88, 259 (1968)

Cope, G. H., Williams, M. A.: Quantitative studies on the preservation of choline and ethanolamine phosphatides during tissue preparation for electron microscopy. I. Glutaraldehyde, osmium tetroxide. Araldite methods. J. Microscopy 90, 31-46 (1969a)

Cope, G. H., Williams, M. A.: Quantitative studies on the preservation of choline and ethanolamine phosphatides during tissue preparation for electron microscopy. II. Other preparative methods. J. Microscopy 90, 47-60 (1969b).

Deamer, D. W., Baskin, R. J.: Ultrastructure of sarcoplasmic reticulum preparations. J. Cell Biol. 42, 296-307 (1969)

Eckert, H.: Distribution of soluble substances at the ultrastructural level. Autoradiography of diffusible substances, p. 321-333. New York: Acad. Press, Inc. 1969

Fiehn, W., Hasselbach, W.: The effect of phospholipase A on the ealcium transport and the role of unsaturated fatty acids in ATPase activity of sarcoplasmic vesicles. Europ. J. Biochem. 13, 510-518 (1970)

Hasselbach, W., Elfvin, L. G.: Structural and chemical asymmetry of the calcium-transporting membranes of the sarcotubular system as revealed by electron microscopy. J. Ultrastruct. Res. 17, 598-622 (1967)

Hasselbach, W., Makinose, M.: Über den Mechanismus des Calciumtransportes durch die Membranen des sarkoplasmatischen Reticulums. Biochem. Z. 339, 94-111 (1963)

Ikemoto, N., Sreter, F. A., Nakamura, A., Gergely, J.: Tryptic digestion and localization of calcium uptake and ATPase activity in fragments of sarcoplasmic reticulum. J. Ultrastruct. Res. 23, 216-232 (1968)

Lengsfeld, A. M., Hasselbach, W.: Struktur and chemische Asymmetrie von Nierenmembranen. Elektronenmikroskopische Untersuchungen an verschiedenen Membranfraktionen des äuBeren Nierencortex (Schwein). Histochemie 27, 253-276 (1971)

Migala, A., Agostini, B., Hasselbach, W.: Tryptic fragmentation of the calcium transport system in the sarcoplasmic reticulum. Z. Naturforsch. 28c, 178-182 (1973)

Mühlethaler, K.: Struktur der Cytomembranen. Arzneimittel-Forsch. 22, 2014-2016 (1972)

Singer, S. J., Nicolson, G. L.: The fluid mosaic model of the structure of cell membranes. Science 175, 720-731 (1972)

Sreter, F., Ikemoto, N., Gergely, J.: The effect of lyophilization and dithiothreitol on vesicles of skeletal and cardiac muscle sarcoplasmic reticulum. Biochim. biophys. Acta (Amst.) 208, 354-357 (1970)

Stein, O., Scow, R. O., Stein, Y.: FFA- ${ }^{3} \mathrm{H}$ uptake by perfused adipose tissue: electron microscopic autoradiographic study. Amer. J. Physiol. 219, 510-518 (1970)

Stein, O., Stein, Y.: Light and electron microscopic radioautography of lipids: Techniques and biological applications. Advances in Lipid Research vol. 9, p. 1-72. New York and London: Acad. Press, Inc. 1971

Stirling, C. E., Kinter, W. B.: High-resolution radioautography of galactose $-{ }^{3} \mathrm{H}$ accumulation in rings of hamster intestine. J. Cell Biol. 35, 585-604 (1967) 
Venable, J. H., Coggeshall, R.: A simplified lead citrate stain for use in electron microscopy. J. Cell Biol. 25, 407-408 (1965)

Wohlfarth-Bottermann, K. E.: Die Kontrastierung tierischer Zellen und Gewebe im Rahmen ihrer elektronenmikroskopischen Untersuchung an ultradünnen Schnitten. Naturwissenschaften 44, 287-288 (1.957)

\author{
Dr. Anneliese Lengsfeld \\ Max-Planck-Institut für med. Forschung \\ Abt. Physiology \\ D-6900 Heidelberg \\ Jahnstraße 29 \\ Federal Republic of Germany
}

